\title{
WIRELESS POWER TRANSFER TO SITALLITE STRATOSPHERIC PLATFORM
}

\author{
Peter J. Schubert ${ }^{1}$, Erinn van Wynsberghe ${ }^{2}$, Abigail J. Kragt Finnell ${ }^{1}$, Cristian Salgueiro ${ }^{1}$, and Ramaa Saket Suri ${ }^{1}$ \\ ${ }^{1}$ Indiana University-Purdue University Indianapolis, Indianapolis, Indiana, U.S.A. pjschube@iupui.edu \\ ${ }^{2}$ VanWyn Aerospace Logistics, Hamilton, Ontario, Canada erinnvw@vanwyn.com
}

Keywords: Pseudo-satellite, WPT, HAPS, stratosphere, safety, design

\begin{abstract}
Stratospheric superpressure balloons are a class of high-altitude pseudo-satellite (HAPS) with excellent observational and communications capabilities. The challenge to long-duration operation is station keeping against the thin but fast zephyrs at altitudes around $25 \mathrm{~km}$. One solution is captured in the US patent 10,404,353, which describes a "Sitallite" HAPS having plasma air thrusters to maneuver against the wind. To hold station for months at a time requires a steady delivery of power, such as a microwave beam transmitted from a terrestrial phased array antenna (PAA) to a rectifying receiving antenna (rectenna) mounted on the underside of the superpressure balloon. This work explores the design and safety considerations for the wireless power transfer (WPT) aspects of such a Sitallite and the tradeoffs between array sizes and power requirements. Presented here are studies of the directivity of the PAA as a function of the number and spacing of antenna elements, and the expected impact on ground personnel, insects, birds, and overflying aircraft. The support and suspension structure of the high-altitude rectenna are developed, along with a comparison of various commercial Schottky diodes for this application. Finally, system studies are presented to illustrate the cost drivers and size optimization. Such a Sitallite can provide line-of-sight visibility for a $1,100 \mathrm{~km}$ radius, remain aloft for many months, and provide services normally expected from an orbiting satellite at a fraction of the cost.
\end{abstract}

\section{INTRODUCTION}

Earth observation and communications are important functions of orbiting satellites, but these are expensive capabilities. In addition to launch costs, there are nonrecurring costs in design for the space environment, integrating with a power bus, packaging into a rocket, securing an orbit, and setting up communications links. Beyond cost is the risk factor of deployment to space including destruction mid-launch, or malfunction while in orbit due to meteors, collisions, radiation, or space debris, without the option for repair or retrieval and thus rendering the investment lost. A lower-cost alternative is a high-altitude pseudo-satellite (HAPS) operating in the stratosphere between the aircraft lanes below and the burn-up of meteorites above. HAPS may be fixed-wing with powered propulsion, or balloon- or blimp-based, either drifting or powered.

The stratosphere is cold, about -40 to $-60{ }^{\circ} \mathrm{C}$, with pressure about 0.01 relative to sea-level, and having winds up to about $30 \mathrm{~m} / \mathrm{s}$. This is much too high for a tether, so a HAPS intended to stay on-station must either carry its own energy source, collect it from sunlight, or have power beamed to it wirelessly [1,2]. This latter solution is the subject of US patent 10,404,353 (2019) by co-author E. van Wynsberghe, which teaches the use of wireless power transfer (WPT) from ground to a HAPS superpressure balloon to power air thrusters for station keeping. Such a system, classified as an Untethered High-Altitude Aerostat (UHAA) and called a "Sitallite," can provide persistent surveillance and communications or Internet links across an area of up to 1 million sq. $\mathrm{km}$.

This work studies the design of a Sitallite WPT system and addresses key tradeoffs and safety considerations. The target application is a stationary location for long-duration missions. The deliverable was a system that could transfer sufficient power such that $30 \mathrm{~kW}$ would be received by the rectenna beneath the Sitallite craft at target altitude. Some costs are omitted due to competitive considerations.

\section{METHODS}

The transmit antenna is a phased array antenna (PAA). One design has elements arranged in a triangular lattice having a hexagonal outline. This configuration has been shown to achieve ultra-low sidelobe levels (e.g., -240 dB) for large arrays [3]. Such arrays less than seven meters in diameter (at $2.45 \mathrm{GHz}$ ) are unable to out-perform traditional PAA arrangements, which have first sidelobe levels (SLLs) of between -60 and $-40 \mathrm{~dB}$ [4]. For considerations of logistics and cost, a smaller PAA is desired; however, the power required of the transmitter increases as the number of elements is reduced. One optimal point minimizes the total cost of both PAA and generators. This study assumes a remote Sitallite location with all power provided by diesel generators. Considerations of safety for passersby, birds, insects, and aircraft are addressed herein. Transmission frequency of $2.45 \mathrm{GHz}$ was selected as a baseline for component heritage and broad acceptability among options in 
the Industrial, Scientific, and Medical (ISM) bands, as regulated by the International Telecommunication Union (ITU).

The rectifying receiving antenna (rectenna) on the Sitallite underside must have an unobstructed view of the transmit antenna, and a small off-axis angle. Furthermore, the plane of the rectenna should be level to avoid destructive interference between DC feeds from diverse RF-to-DC rectifier elements. The rectenna must be stiff, but also strong, and should avoid resonant frequencies that might get excited by the stratospheric wind. A suspension system consists of a net or strap system around the top surface of the superpressure balloon, from which the rectenna plane is hung. Power management and distribution devices are located at the center for symmetry, delivering power to the payloads located at the periphery of the rectenna or Sitallite, whichever is wider. The thrusters are powered during ascent and descent for safety.

\section{RESULTS}

The one-line diagram of the transmitter subsystem is shown in Fig. 1, which shows the use of an AC diesel generator driving a power amplifier, which boosts an RF oscillator signal. Phase shifters modulate the phase angle between elements to effect an electronically-steered beam.

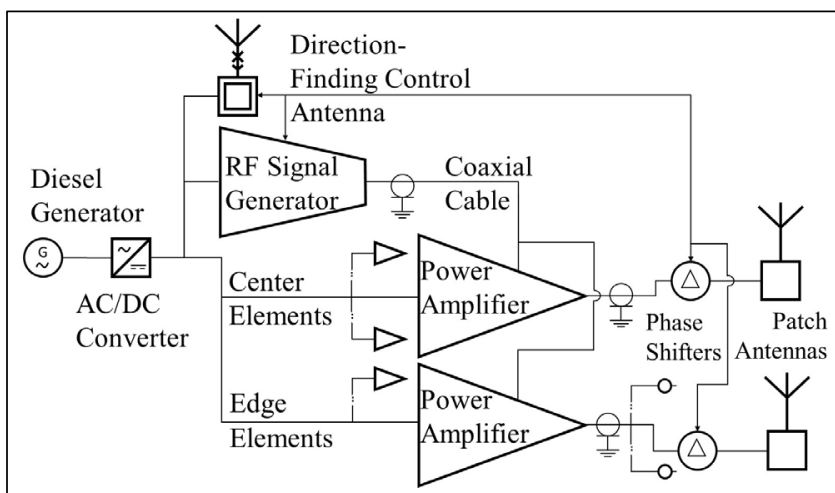

Figure 1. One-line diagram of transmitter circuit.

Directivity is the maximum proportion of signal in a given direction. For a PAA, the directivity increases linearly with the number of elements (see Fig. 2). This data was generated using AWR software.

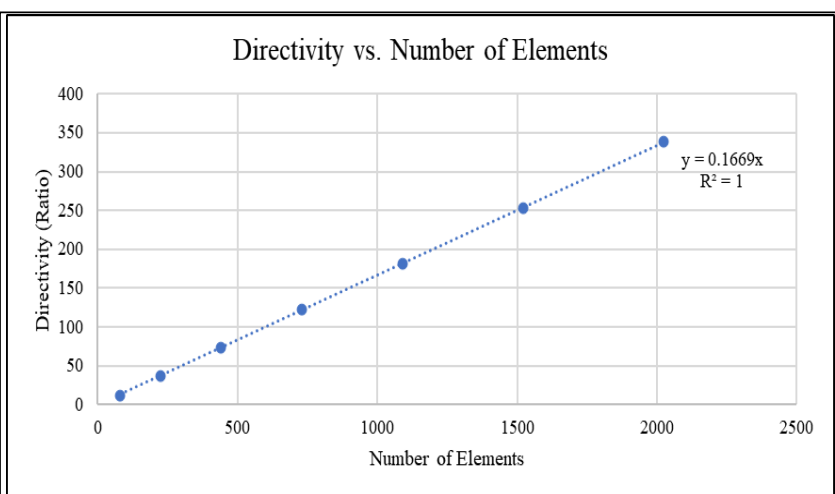

Figure 2. Directivity as a function of elements in a PAA.

With smaller PAA having fewer elements and shorter side length (assumed square for convenience), with lower directivity, the power required increases significantly. Smaller PAA, therefore, demand greater power from each element and have lower overall efficiency.

Fig. 3 shows the relation of total power input at the transmitter as a function of transmitter size for three sizes of (circular) rectenna at $25 \mathrm{~km}$ altitude. The power collection efficiency at the receiver is assumed to be $65 \%$. A nominal rectenna diameter of 80 meters is selected as a baseline: it is small enough that it can be contained within the anticipated diameter and lifting capabilities of an optimal superpressure balloon with minimal drag, but still large enough that it will help ensure a comparatively small transmitter size on ground which comes with smaller component costs (discussed below), smaller footprint and land costs, and easier setup and take-down. Additionally, a smaller transmitter could take up less deck space aboard an aircraft carrier and so could enable ocean-based applications. A larger passive rectenna is more cost-effective than a larger power transmitter. Fig. 3 reveals that the amount of power transmitted, and hence the number of generators, decreases as the PAA is made larger.

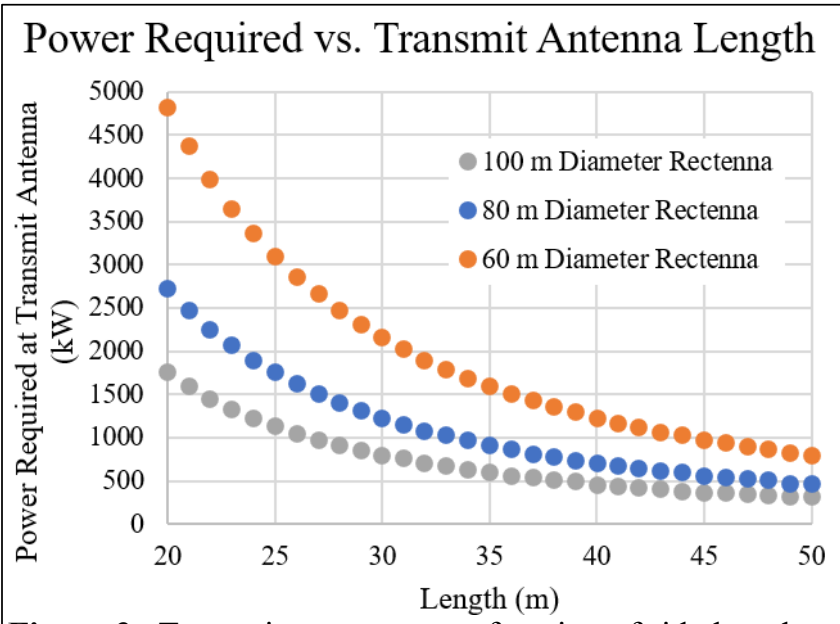

Figure 3. Transmitter power as a function of side length and rectenna diameter at $25 \mathrm{~km}$ altitude. 
However, a larger PAA size increases the number of components and hence the cost. Components include power amplifiers, phase shifters, patch antennas, circuit boards, power cables, connectors, RF oscillator, ground screws for mounting, and structural members to support the PAA above the Earth. The relative proportion of these components, plus the cost of generators and the yearly cost of diesel fuel, is shown in Fig. 4. Note that if the gensets are replaced by grid electricity, the cost comes down significantly, although the cost of electricity still dominates at $39 \%$ of the total transmitter cost.

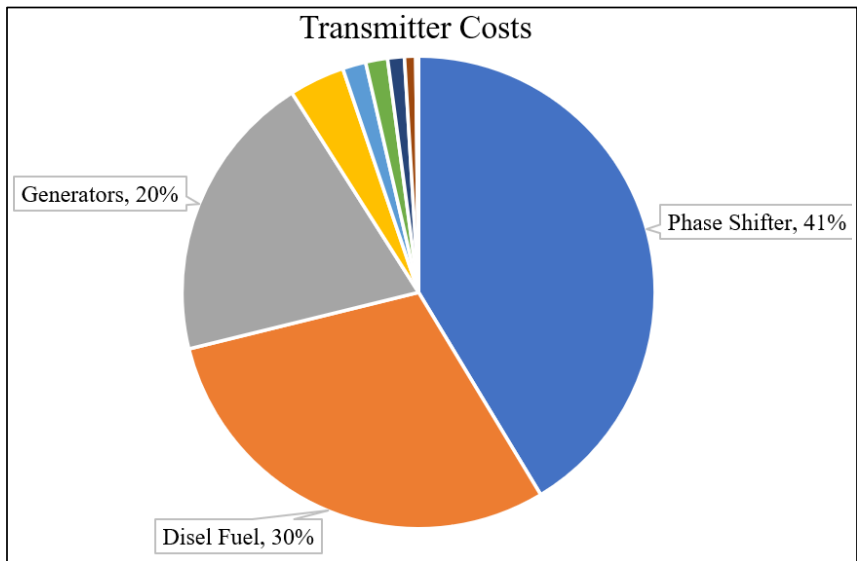

Figure 4. Cost breakdown for a remote PAA transmitter location, half of which can be eliminated with a power grid.

Fig. 5 illustrates the overall optimum cost for a remote location using diesel gensets to power a ground-mounted PAA transmit antenna delivering power wirelessly to an $80 \mathrm{~m}$ diameter rectenna at $25 \mathrm{~km}$ altitude. The total cost for a remote installation is the topmost line, with the minimum cost shown with a red dot, this being at a $35 \mathrm{~m}$ PAA side length.

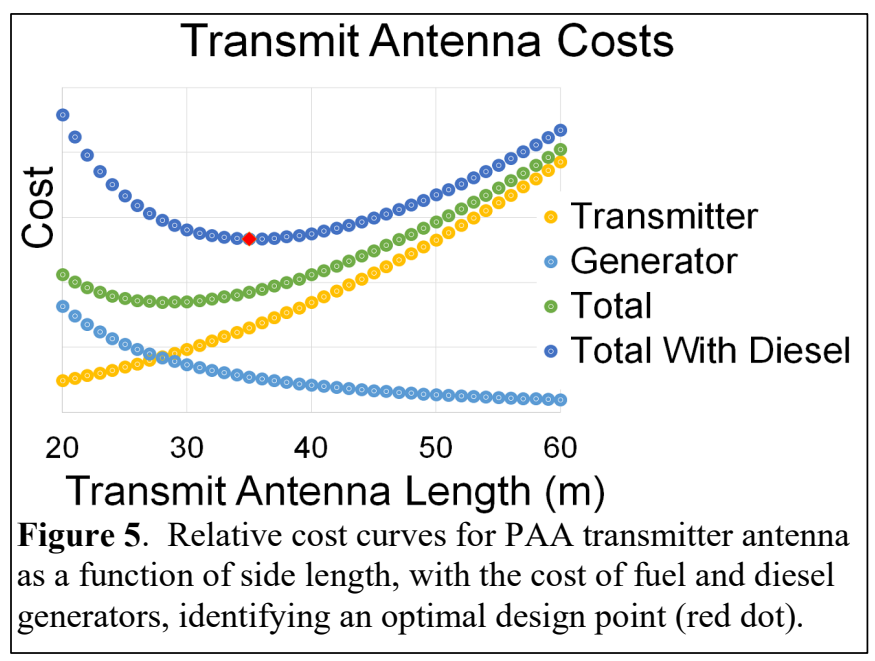

A cross-sectional schematic of the Sitallite is shown in Fig. 6. At the bottom is the rectenna structure, above which is a microwave absorber to prevent excessive heating of the mechanical structure and superpressure balloon material. At right are the controls and payload, shown extending past the edge of the rectenna so as to avoid signal interference with the power beam. At left is shown energy storage, such as a battery (heated), which may be required to provide power to the thrusters (red squares) should the transmit beam blink out, or is poorly-aligned such as during ascent and descent.

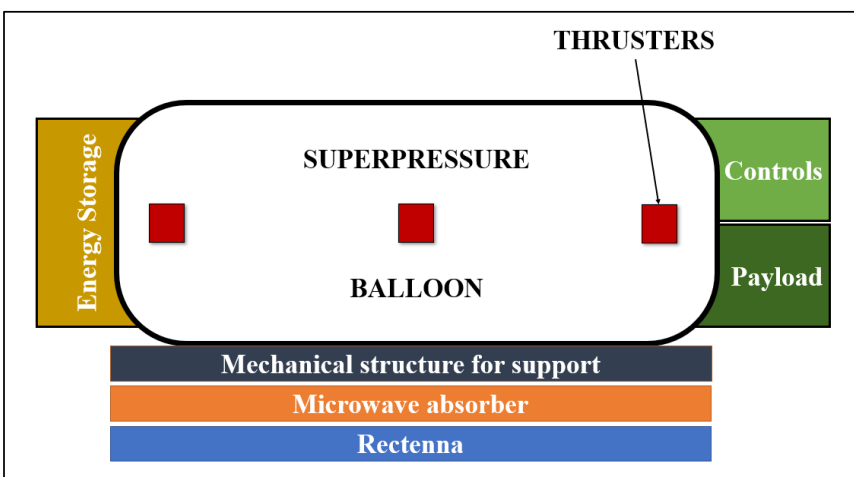

Figure 6. Cross-section schematic of Sitallite platform.

In order to receive power wirelessly aboard the craft, the rectifying antenna (rectenna) must be designed such that each panel can effectively manage the power load it receives. Panels closer to the center of the beam will be subject to greater energy density than panels toward the periphery. The rectifying circuits for each panel were designed based on the work of W.C. Brown, whose circuit designs employed shunt diodes. Design work began with sourcing which diodes were accessible in industrial quantity. Several commerciallyavailable diodes were considered, and evaluated on the basis of five variables, namely, forward on voltage $\left(\mathrm{V}_{\mathrm{f}}\right)$, reverse breakdown voltage $\left(\mathrm{V}_{\mathrm{BR}}\right)$, on-resistance $\left(\mathrm{R}_{\mathrm{S}}\right)$, junction capacitance $\left(\mathrm{C}_{\mathrm{JO}}\right)$, and maximum frequency $\left(\mathrm{f}_{\max }\right)$. A spider chart of this analysis is shown in Fig. 7. Using the Brown rectenna configuration with a shunt diode across the antenna leads [5], the efficiency of the rectenna is optimized at higher power densities by the difference between $V_{f}$ and $V_{B R}$, and the quality of the output DC depends on the capacitance [6]. The Schottky diode YSD110 appears to offer a good tradeoff in performance. Fig. 8 shows the efficiency as a function of input power to an individual rectenna element. This data was based on a $200 \mathrm{Ohm}$ resistive load and a $5 \mathrm{pF}$ smoothing capacitor. 


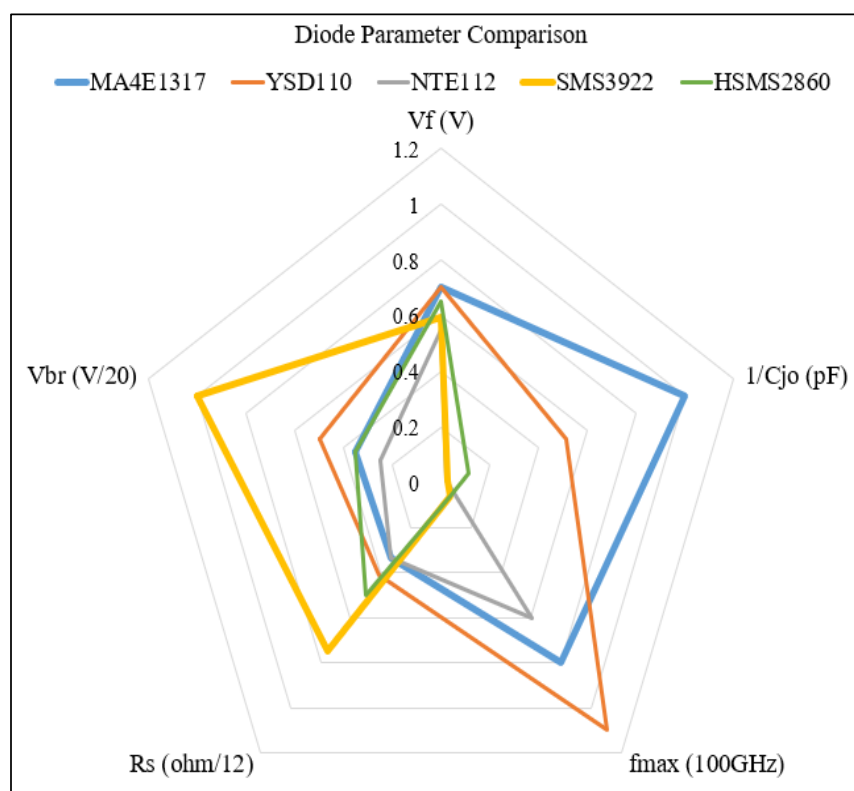

Figure 7. Schottky diode comparison for rectenna circuit.

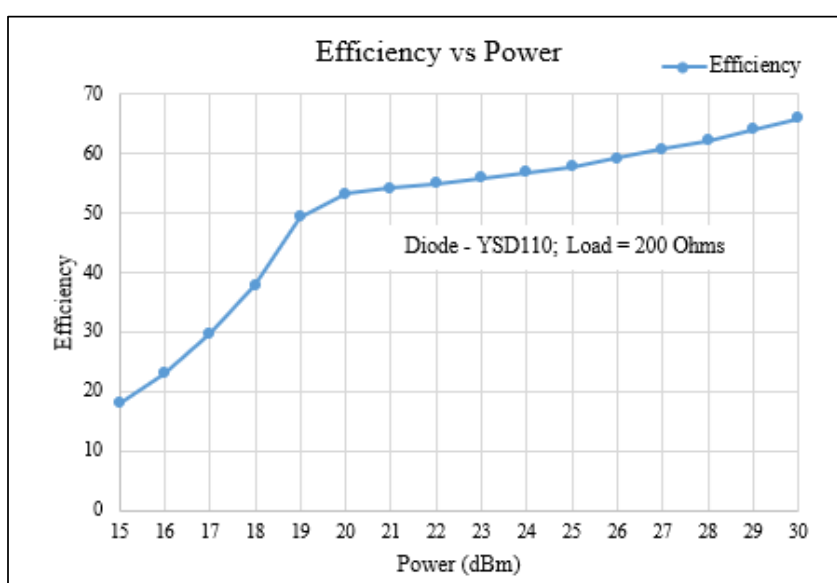

Figure 8. Rectifier efficiency versus input power at $200 \Omega$.

Fig. 9 shows the rectenna costs as a function of diameter. The cost rises faster than linearly because of the larger number of components with larger area, and also because of the increased demands for structural support. There is a tradeoff between transmit antenna size and Sitallite rectenna size. On the basis of this graph, plus the results in Fig. 3, the rectenna size was fixed at 80 meters, receiving power from a transmitter sized at 35 meters.

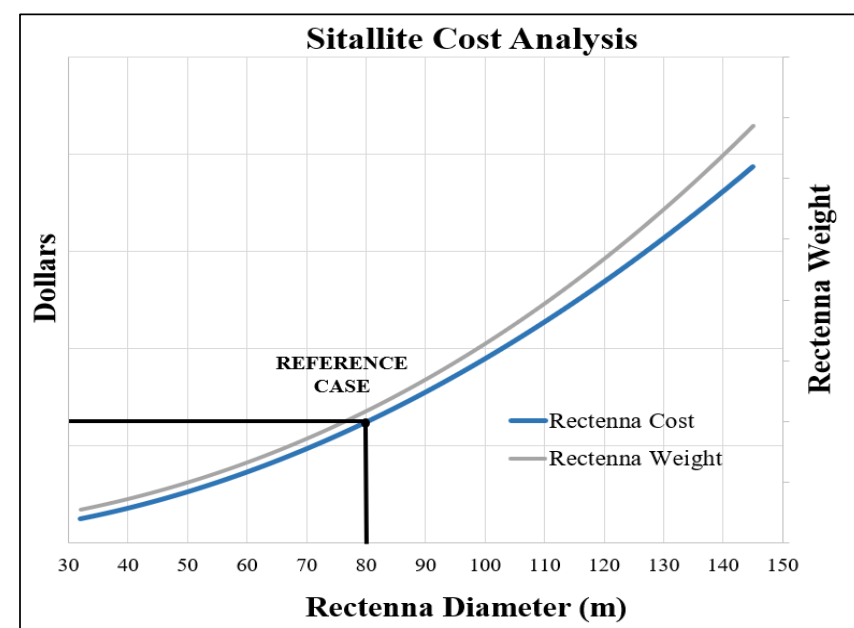

Figure 9. Supra-linear cost, weight with rectenna size.

\section{SAFETY}

Two factors minimize the level of exposure to ground workers while the transmit antenna is operating. First, the high directivity means that most of the energy is going straight up to the Sitallite. Second, the power density profile of the PAA has greatly reduced amplitude at the periphery, as much as three orders of magnitude lower than at the center. The energy emitted by each antenna element is quite small. Thus, the amount of RF energy directed laterally from the PAA is minimal. Table 1 shows the exposure to birds, and those aircraft which may fly through the power beam. The FCC Limit for Maximum Permissible Exposure is $1 \mathrm{~mW} / \mathrm{cm}^{2}$ over 30 minutes [7]. If time averaged, all power densities would be equivalent to less than $0.1 \mathrm{~mW} / \mathrm{cm}^{2}$.

\begin{tabular}{|l|l|l|l|}
\hline Item & $\begin{array}{l}\text { Typical } \\
\text { Height }(\mathrm{km})\end{array}$ & $\begin{array}{l}\text { Power } \\
\text { Density } \\
\left(\mathrm{mW} / \mathrm{cm}^{2}\right)\end{array}$ & $\begin{array}{l}\text { Time } \\
\text { Exposed } \\
(\mathrm{s})\end{array}$ \\
\hline Canada Goose & 1 & 606 & 0.29 \\
\hline Small Airplane & 4 & 82 & 0.33 \\
\hline $\begin{array}{l}\text { Commercial } \\
\text { Airplane }\end{array}$ & 9.5 & 14.7 & 0.19 \\
\hline
\end{tabular}

Table 1. Safety considerations for airborne passersby.

\section{FUTURE WORK AND OPPORTUNITIES}

The Sitallite concept will require additional development work including rigid platform to support the rectenna, optimization of thrusters and balloon for aerodynamic performance at target altitude, integration of the above with supporting components including scaffold, sensors, controls, radio, payload, and power distribution (along with corresponding thermal management, wiring, and containers), testing at successive scales, and all appropriate safety and regulatory affirmations. 
Wireless power transfer from ground represents significant economic opportunities including beaming power to any and all electric aircraft for indefinite flight; removal of fossil fuel use from the skies; remote power to satellites, orbital telescopes, and other space assets; space debris detection and removal; beamed power propulsion; and much more.

For the Sitallite in particular, power sent from ground offers advantages over power sent from space, namely shorter transmission distance $(25 \mathrm{~km}$ vs hundreds for low earth orbit and tens of thousands for geosynchronous or geostationary orbit). Shorter distance offers lower transmission losses, reduced design complexity, and smaller and fewer components, which reduces cost. The absence of any space launch requirement provides even greater advantages including vastly lower cost, risk, complexity, and time delay, along with greater control of assets.

Fortuitously, ground-based power-beaming can serve as a valuable stepping stone toward the development of larger space-borne WPT projects including transmitting solarharvested power from orbit down to Earth (space-based solar power), along with remote power at the moon, asteroids, planets, and other space bodies as well as future satellites, stations, spacecraft, and other assets to facilitate new markets of tourism, mining, colonization, and exploration. The near-term development of ground-based power transfer, in addition to creating revenue-generating products and services, can also advance state-of-the-art, validate the capability of WPT to non-technical audiences and prospective customers, and engage the general public and future generations of STEM professionals in a now-tangible industry of power beaming.

\section{CONCLUSIONS}

The Sitallite is a patented HAPS concept which uses WPT to great advantage in providing long-duration, persistent surveillance and data links. With a reference case of a circular rectenna of 80 meters diameter beneath the craft at target altitude, a square transmitter of length of $35 \mathrm{~m}$ was calculated to be of optimal value, weighing factors of power consumption and cost of parts. By using plasma air thrusters, there is no fuel consumption during ascent, descent and while keeping the Sitallite stationed in the atmosphere. With beamed power, there is no need for fuel, and minimal need for on-board energy storage. There is an optimal size for remote installations, and the overall costs are much lower than for an orbiting satellite. Future work will center on the rectenna structural design, plasma air thrusters, and control system. WPT from ground offers advantages over power from space, but also represents a stepping-stone opportunity to advance such projects including space-based solar power.

\section{ACKNOWLEDGEMENT}

This work was made possible by funding from the Canadian Department of National Defense through the Innovation for Defence Excellence and Security (IDEaS) program, and by VanWyn Aerospace Logistics.

\section{REFERENCES}

1 A. Massa, G. Oliveri, F. Viani, and P. Rocca, "Array designs for long-distance wireless power transmission: State-of-the-art and innovative solutions," Proceedings of the IEEE, vol. 101, no. 6, pp. 1464-1481, 2013.

2 E. van Wynsberghe, and A. Turak, "Station-keeping of a high-altitude balloon with electric propulsion and wireless power transmission: A concept study," Acta Astronautica. 128, 2016., doi: d10.1016/j.actaastro.2016.08.017.

3 P.J. Schubert, "Sidelobe reduction for GEO to earth wireless power transfer," in 67th International Astronautical Congress (IAC 2016), 2016.

4 A.J. Finnell, P.J. Schubert, "Antenna Arrangement Verification for Low Sidelobe Levels," WiSEE 2019, Ottawa, Ontario, Canada, Oct 2019.

5 W.C. Brown, "The History of the Development of the Rectenna.[solar power satellites]." (1980).

6 J.O. McSpadden, Lu Fan, and Kai Chang. "Design and experiments of a high-conversion-efficiency $5.8-\mathrm{GHz}$ rectenna." IEEE Transactions on Microwave Theory and Techniques 46.12 (1998): 2053-2060.

7 J.L. Ulcek, and R.F. Cleveland Jr., "Evaluating Compliance with FCC Guidelines for Human Exposure to Radiofrequency Electromagnetic Fields", Supplement B to OET Bulletin 65 Edition 97-01, 1997. 